\title{
Design of a detector to study $S=-2$ baryon interactions induced by stopped antiproton annihilation
}

\author{
D. Alfs ${ }^{1,2, *}$, D. Grzonka ${ }^{1}$, K. Kilian ${ }^{1}$, J. Ritman ${ }^{1}$, and T. Sefzick ${ }^{1}$ \\ ${ }^{1}$ Institut für Kernphysik, Forschungszentrum Jülich, Jülich, Germany \\ ${ }^{2}$ M. Smoluchowski Institute of Physics, Jagiellonian University, Kraków, Poland
}

\begin{abstract}
Stopped antiproton annihilation can initiate an efficient chain of reactions to produce $\Xi$ hyperons in recoil free kinematics, suitable for further investigations of $\Xi N$ interactions. Four delayed decays and presence of eight charged particles in the final state provide a clean reaction signature and allow for usage of a non-magnetic detection system. With the low energy phase space cooled antiproton beam from ELENA at the CERN AD or future FLAIR facility the realisation of this experiment would become possible. In this article the foreseen detector setup and the experimental program will be presented.
\end{abstract}

\section{Motivation}

The experimental determination of the baryon-baryon interaction within the baryon octett allows for development of improved hyperon-nucleon potentials and investigation of SU(3) flavor symmetry. While for the $N N$-system an extended data base exists, the hyperon sector is much less explored and the studies of strangeness $S=-2$ systems are practically limited to searches for the H-particle [1, 2].

Conventionally, the $\Xi$ production proceeds via a double strangeness exchange reaction $(s, \bar{s})$ induced by a secondary $K^{-}$beam, e.g. $K^{-} p \rightarrow \Xi^{-} K^{+}[3]$. In this case $\Xi$ production in recoil-free kinematics is not feasible since the mass of $K^{-}$is too low and a secondary target would be needed for the $\Xi$ to undergo hadronic interactions.

A much more efficient approach would be a direct production of slow $\Xi$ hyperons with momenta down to zero [4]. The proposed reaction chain starts with $K^{*}$ produced in stopped antiproton annihilation. The momenta of the $K^{*}$ produced this way are well matched to the momentum range required to produce slow cascades which can go further into interacting $\Xi N$ systems.

\section{Production of $S=-2$ systems via double strangeness exchange reaction}

A $K^{*}$ 'beam' is produced in the antiproton annihilation reactions on a nucleon. The production of $S=-2$ systems proceeds in a second step via the double strangeness and charge exchange reaction $\left(K^{*}, K\right)$. Due to the $K^{*}$ decay length of a few fm, both the production of

\footnotetext{
*e-mail: d.alfs@fz-juelich.de
} 
the $K^{*}$ and the double strangness and charge exchange reaction must take place in the same nucleus.

The exchange reactions can proceed via $\bar{K}^{*} N \rightarrow K \Xi$, e.g. $K^{*-} p \rightarrow K^{+} \Xi$. From the reaction kinematics, a certain $K^{*}$ momentum results in $\Xi$ production with very low momentum in the laboratory frame, i.e. for the reaction $\bar{p} p \rightarrow \bar{K}^{*} K^{*}\left(m_{K^{*}}=982 \mathrm{MeV} / \mathrm{c}^{2}\right)$ momentum of $K^{*}$ from the annihilation for the nominal $m_{K^{*}}$ is equal to about $290 \mathrm{MeV} / \mathrm{c}$, while the $K^{*}$ momentum appropriate for the $\Xi$ recoil-free production is $160 \mathrm{MeV} / \mathrm{c}$. Considering that the $K^{*}$ has a width of about $50 \mathrm{MeV}$, the conditions for recoil free production are rather well matched.

The first step of the studies would be the investigation of the branching ratio of the $\Xi$ production in the stopped $\bar{p}$ annihilation in a deuteron target. For the further study of $\Xi N$ systems interaction, ${ }^{3} \mathrm{He}$ target is needed.

\section{Detection principle}

An exemplary reaction chain considered for the purpose of the detection setup description is $\bar{p} d \rightarrow \Xi^{-} K_{S}^{0} K^{*+} \rightarrow\left(p \pi^{-}\right)_{\Lambda} \pi^{-}\left(\pi^{+} \pi^{-}\right)_{K_{S}}\left(\pi^{+} \pi^{-}\right)_{K_{S}} \pi^{+}$(see Fig. 1) [4, 5]. A selective trigger for this reaction is the increase of the charged particles multiplicity (due to four delayed decays of strange neutral particles): from multiplicity equal to two close to the target up to eight in the final state.

The reaction identification is possible via the analysis of the geometry of charged particle tracks: from the charged pions the decay vertices of the $K_{S}^{0}$ can be reconstructed and, with a known target position, their tracks are determined. Then, the $\Lambda$ track is determined from the $\left(p, \pi^{-}\right)$and the $\left(\Xi^{-}, \pi^{-}\right)$tracks. This allows for a full kinematic event reconstruction. The interaction point is provided by the cascade track and the prompt pion from $K^{*}$ decay. Furthermore the three $K_{S}^{0}$ decays provide decay planes and their intersection lines give a further definition of the (largely overdetermined) interaction point.

For the reaction on a ${ }^{3} \mathrm{He}$ target, the expected configuration of the particles in the final state is similar, with one additional proton in the exit channel.

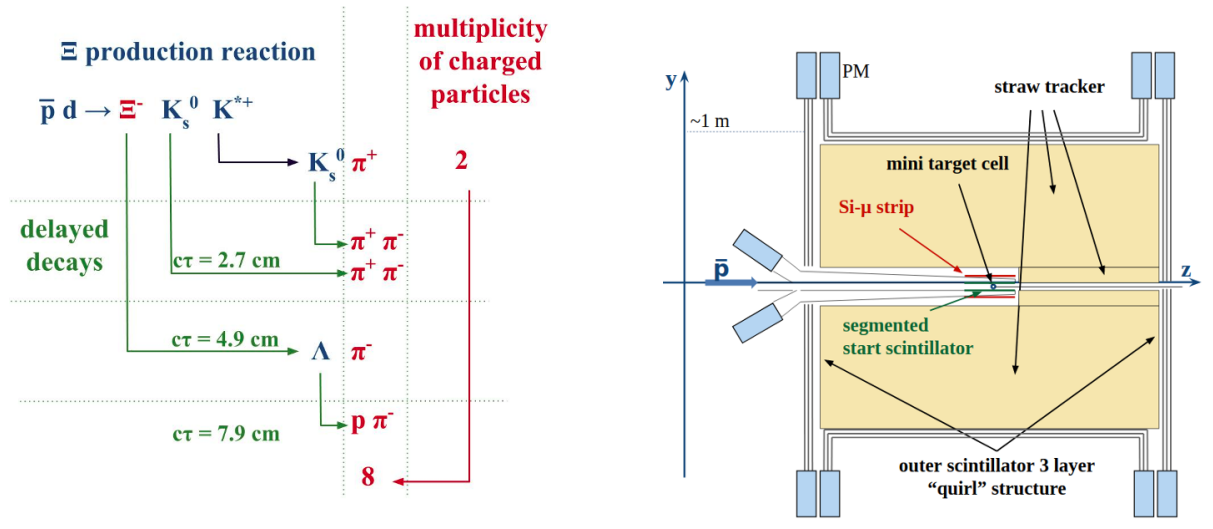

Figure 1. Left: Production of $\Xi$ hyperon - an exemplary reaction channel. Right: a scheme of the proposed detector.

The proposed experimental setup is shown in Fig. 1. Due to the expected high multiplicity of charged particles, the detector geometry needs to be close to $4 \pi$. A vertex detector (e.g. Si- $\mu$ strips) for the detection of particles with a very short $c \tau$ (e.g. $\Xi^{-}$from the exemplary reaction). 
For further three dimensional tracking a straw tube detector is foreseen. The final layout still needs to be validated by Monte Carlo studies. The outermost part of the detector (a barrel type together with endcaps) may be plastic scintillator hodoscopes.

A very small high pressure gaseous target within a thin-walled container and a phase space cooled low emittance antiproton beam (with energy of about $1 \mathrm{MeV}$ ) is required. According to exemplary GEANT4 MC studies of $0.7 \mathrm{MeV}$ antiproton beam $\left(\sigma_{\mathrm{x}, \mathrm{y}}=1 \mathrm{~mm}\right)$ hitting a gaseous ${ }^{3} \mathrm{He}$ target cell $(\mathrm{T}=20 \mathrm{~K}, \mathrm{p}=1 \mathrm{bar}, \mathrm{r}=2.5 \mathrm{~mm})$ with a $0.01 \mathrm{~mm}$ Mylar foil window, less than $5 \mathrm{~mm}$ is enough to stop the antiprotons (see Fig. 2). In the final design the target cell could be a small sphere of a few mm diameter at the end of a less than $1 \mathrm{~mm}$ diameter tube.

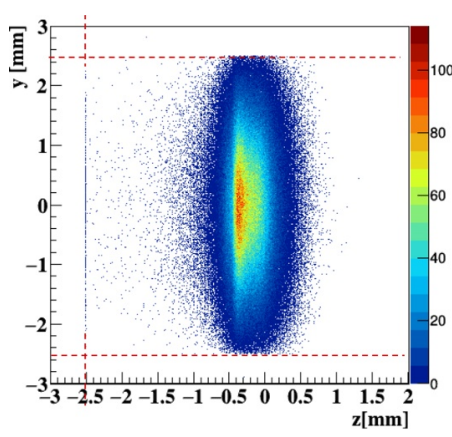

Figure 2. Results of GEANT4 Monte Carlo simulations (Physics List: QGSP_BERT) of a $0.7 \mathrm{MeV}$ antiproton beam $\left(\sigma_{\mathrm{x}, \mathrm{y}}=1 \mathrm{~mm}\right)$ stopping in a ${ }^{3} \mathrm{He}$ target cell $(\mathrm{T}=20 \mathrm{~K}$, $\mathrm{p}=1 \mathrm{bar}, \mathrm{r}=2.5 \mathrm{~mm}$ ) with a $0.01 \mathrm{~mm}$ Mylar foil window (visible at $-2.5 \mathrm{~mm}$ ). The target volume is indicated with red dashed lines. From the technical point of view, it might be more beneficial to operate the target cell at higher gas pressures and higher temperatures. For the final conditions further simulations and experimental studies are required.

\section{Summary and perspectives}

A reaction chain resulting in the production of a $\Xi$ hyperon with a low recoil momentum down to zero $\mathrm{MeV} / \mathrm{c}$ enabling investigations of $\Xi N$ interactions was proposed based on ideas already proposed for LEAR [4]. Due to the presence of four delayed decays the reaction can be clearly identified online. The proposed detector setup is currently designed with a focus on a target cell and Monte Carlo studies of low energy antiproton stopping in the gaseous media.

The low energy phase space cooled antiproton beam which will be available from ELENA [6] or FLAIR [7]. However, these studies require a slow extracted antiproton beam which will be available at FLAIR but is currently not delivered from ELENA. A possible solution could be trapping of antiprotons and subsequent slow extraction from the trap but this would require a more detailed consideration for future realization.

Acknowledgements: This work was supported by Marie Skłodowska-Curie Innovative Training Network Fellowship of the European Commission's Horizon 2020 Programme (No. 721559 AVA).

\section{References}

[1] R.L. Jaffe, Phys. Rev. Lett. 38, 195 (1977)

[2] T. Sakai, K. Shimizu, K. Yazaki, Prog. Theor. Phys. Suppl., 137, 121 (2000)

[3] K. Nakazawa, Nucl. Phys. A, 639, 345 (1998)

[4] K. Kilian, Proc. 4th LEAR Workshop, Villars-sur-Ollon, Switzerland, 1987, ed. C. Amsler et al., (Harwood Academic Publishers Chur London, UK, 1988) 529

[5] D. Grzonka et al., AIP Conf. Proc. 793190 (2005)

[6] T. Eriksson, Proceedings of IPAC2017, Copenhagen, Denmark, 2017, ed. V. Schaa et al., (JACoW, Geneva, Switzerland, 2017) 3327

[7] C.P. Welsch, Proceedings of the 4th International Conference on Trapped Charged Particles and Fundamental Physics (TCP 2006), ed. J. Dilling et al., (Springer, Berlin, Heidelberg, 2007) 71 